\title{
Metallic Foreign Body in the Liver of a Dog
}

\author{
Hojung CHOI ${ }^{1)}$, Youngwon LEE $^{1)}$, Jiwhan $\mathrm{WANG}^{2)}$, Seongchan YEON ${ }^{2)}$, Hyojong LEE ${ }^{2)}$ and Heechun LEE $^{2) *}$ \\ ${ }^{1)}$ Department of Veterinary Medical Imaging, College of Veterinary Medicine, Chungnam National University, Daejeon and \\ ${ }^{2}$ Research Institute of Life Sciences, Gyeongsang National University, Jinju, South Korea
}

(Received 15 March 2010/Accepted 4 June 2010/Published online in J-STAGE 18 June 2010)

ABSTRACT. A 4-year-old beagle had intermittent vomiting and weight loss for 1 month. On plain radiography, an approximately 3.5-cm, radiopaque, linear foreign body was identified in the region of the liver. On ultrasonography, a hyperechoic linear structure with reverberation was identified in the left lobe or quadrate lobe. On computed tomography(CT), a thin hyperattenuating material consistent with a metallic foreign body was identified just medial to the gall bladder. Removal of the foreign body was performed without complications. We present a rare case in which a metallic foreign body was embedded in the liver asymptomatically and found incidentally during a clinical examination performed to ascertain the cause of clinical signs.

KEY WORDS: eight loss, metallic foreign body, reverberation.

J. Vet. Med. Sci. 72(11): 1487-1490, 2010

Foreign body migration represents a sufficiently documented entity in veterinary and human literature. In the dog, migrating foreign bodies involve grass awns, wood fragments, wooden skewers, needles and toothpicks, and these foreign bodies migrate to multiple body regions including the eyes, vertebrae, urinary tract and abdominal and thoracic cavities, resulting in various clinical syndromes $[1,5-8,11$, 12, 19].

A 4-year-old, 9.3-kg, male beagle with no previous history of related medical problems or surgery was presented to Gyeongsang National University Veterinary Teaching Hospital with a 1-month history of intermittent vomiting and weight loss (about $3 \mathrm{~kg}$ ). There was also a 3-day history of anorexia and lethargy. All vaccinations were completed, and the dog was kept outdoors. There was a history of eating expired diet, and the clinical signs had developed since then.

On physical examination, the dog's temperature, pulse, respiratory rate and capillary refill time were normal. Mild dehydration was indicated. The dog had a lean body condition, but its abdomen was not painful upon palpation. Parasitic infection could be ruled out on fecal examination. Its CBC revealed leukocytosis $\left(20.1 \times 10^{3} / \mu l\right.$; reference range, 6.7 to $18.3 \times 10^{3} / \mu l$ ) characterized by mature neutrophilia $\left(14.2 \times 10^{3} / \mu l\right.$; reference range, 3.6 to $\left.12.5 \times 10^{3} / \mu l\right)$ and monocytosis $\left(4.0 \times 10^{3} / \mu l\right.$; reference range, 0.1 to $1.5 \times 10^{3} /$ $\mu l)$ consistent with a stress leukogram. Its erythrogram was unremarkable. Abnormalities in its serum biochemistry profile included hypoalbuminemia $(1.5 \mathrm{~g} / \mathrm{d} l$, normal range: 2.5-4.4) and hypokalemia (2.9 $\mathrm{mEq} / l$, normal range: $3.7-$ 5.8).

Thoracic plain radiography was negative for any abnormalities. However, abdominal plain radiography revealed poor abdominal detail because of the dog's emaciated body

\footnotetext{
* Correspondence to: Lee, H., Research Institute of Life Sciences, Gyeongsang National University \#900, Gajwa-dong, Jinju, Gyeongnam 660-701, South Korea.

e-mail: lhc@gnu.ac.kr
}

condition. In addition, an approximately $3.5-\mathrm{cm}$, radiopaque, linear foreign body was identified in the region of the liver (Fig. 1)

On ultrasonographic images, a hyperechoic linear structure with a reverberation artifact was identified in the liver (Fig. 2). Abdominal non-contrast CT images revealed a thin hyperattenuating material with a high contrast artifact just medial to the gall bladder, and this site was considered to be the quadrate lobe (Fig. 3). Hepatic lesions such as abscess, hematoma, necrosis and gall bladder rupture were not identified around the foreign body on ultrasonographic images nor CT images. The other abdominal organs were unremarkable, including the kidneys, spleen, adrenal glands, small intestine, pancreas and urinary bladder.

Symptomatic therapy for vomiting was performed. The owner was told to cease feeding expired diet and was recommended to have the dog rechecked regularly before clinical signs associated with hepatic foreign body develop. The dog made a full recovery, with no recurrence of the clinical signs by 1 month post-diagnosis. However, due to the possibility of gall bladder rupture, laparotomy was performed, and adhesion was found between the gall bladder wall and medial aspect of the quadrate lobe (Fig. 4A). After dividing the space between the gall bladder wall and medial aspect of the quadrate lobe, the tip of the metallic foreign body was palpated. The metallic foreign body was extracted from the liver by pressing the surface of the liver with mosquito forceps (Fig. 4B). No bleeding or other complications occurred after extraction of the metallic foreign body. The post-operative course was uneventful, and the dog was discharged one week later. Approximately 3 months after discharge, $\mathrm{CBC}$, serum-biochemistry and ultrasound were performed, and all results were normal.

In humans, approximately $80-90 \%$ of ingested foreign bodies entering the stomach pass uneventfully through the gastrointestinal tract within a week without being discovered $[3,9,13,16]$. However, an ingested foreign body may cause hemorrhage, perforation or obstruction. Gastrointes- 

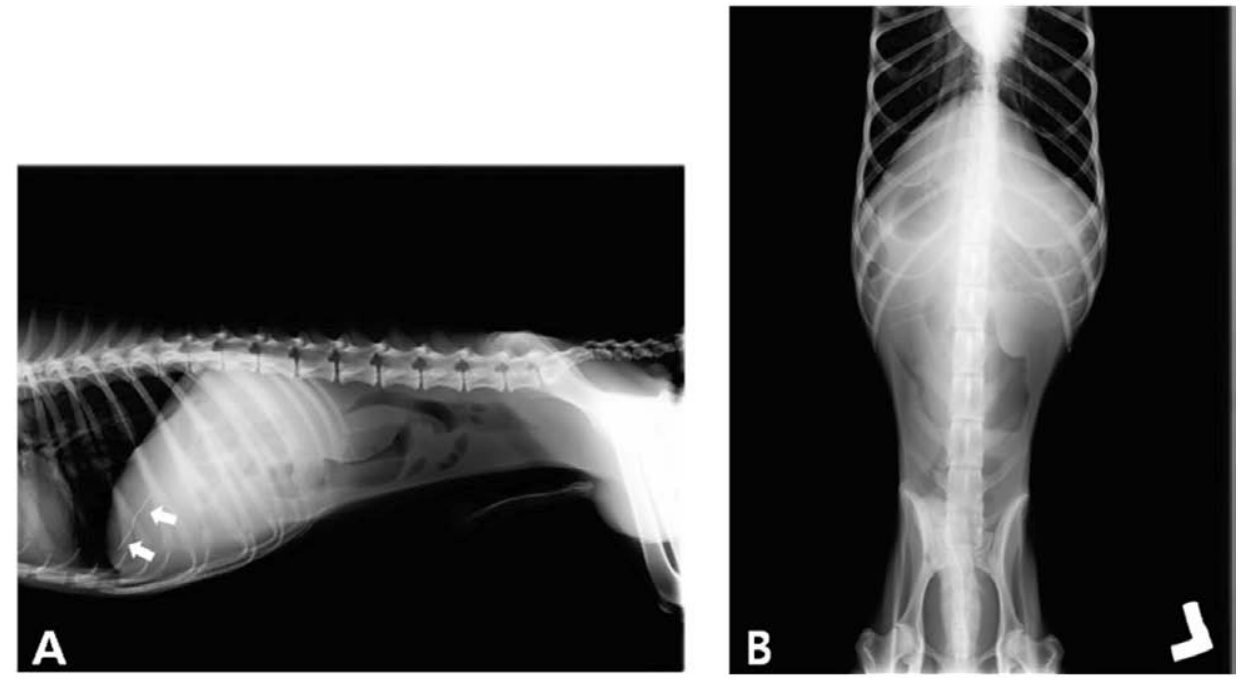

Fig. 1. The lateral (A) radiograph shows the metallic foreign body (arrows) in the region of the liver. This metallic foreign body could not be identified on the ventrodorsal radiograph (B) because it was superimposed over vertebrae. In addition, a uniform, homogeneous, soft tissue opacity was identified throughout the abdomen.

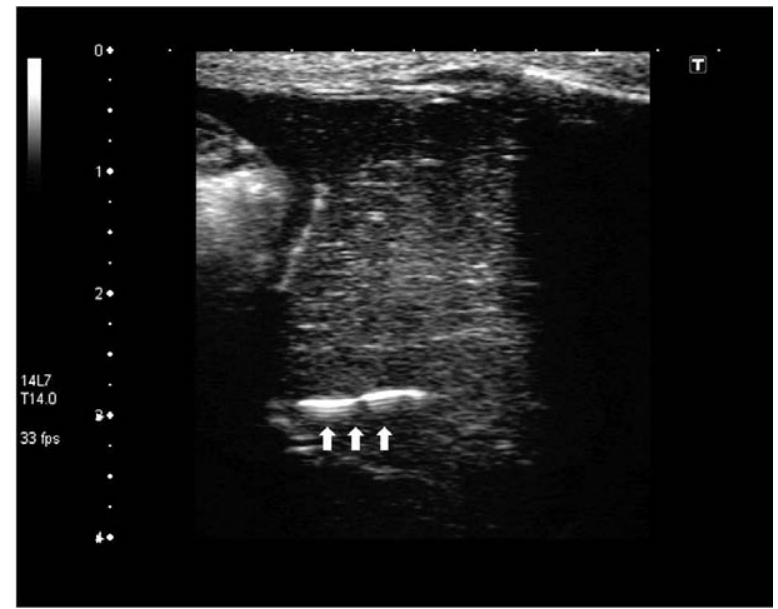

Fig. 2. Longitudinal sonogram of the cranial abdomen. A hyperechoic linear structure with weak reverberation (arrows) was identified in the left lobe of the liver.

tinal perforation has been reported in less than $1 \%$ of patients with long, thin and sharp foreign bodies, such as chicken and fish bones, toothpicks and needles, pens, toothbrushes and dental plates $[3,10,16]$. Forty-six cases of foreign bodies penetrating the liver have been reported worldwide, and hepatic abscess developed in all of the cases. In these previous reports, the foreign bodies included fish bones, chicken bones, needles or toothpicks and pens or dental plates, and the principal origin of migration to the liver was the duodenum or stomach [16]. In the present case, although the type of foreign body in the liver was not ascertained accurately, wire was suspected strongly based on its appearance, and the site of perforation was unknown.
We believed that the principal origin of migration to the liver was the pyloric area, considering the position of the foreign body in the liver. This patient represents a unique case of asymptomatic metallic foreign body migration into the liver.

In the present case, vomiting and weight loss were the chief complaints, and these clinical signs were not specific. As the cause of the clinical signs in this case, the possibility of dietary indiscretion rather than foreign body in the liver was high because, as mentioned previously, no hepatic abnormality was observed on clinical examinations including physical examination, blood screen tests, ultrasound and CT scan. In addition, the dog recovered by changing its diet without specific medical treatment. A migrating foreign body remains asymptomatic for a long time if there are no features of infection or abscess formation $[3,10,16]$.

In the present case, hypoalbuminemia was identified. Although the cause of hypoalbuminemia was not certain, we believe that it was associated with malnutrition or malabsorption due to a dietary indiscretion.

In diagnosis of an ingested foreign body, methods of foreign body detection include radiography, ultrasound, CT, MRI, upper gastrointestinal tract endoscopy, colonoscopy and laparotomy $[2,10,16]$. Radiopaque foreign body may be identified with plain abdominal radiography. However, although a foreign body has bony opacity, plain radiography can be ambiguous because of the masking effect of the soft tissue mass, fluid collection around the penetrated bone and the absence of free gas in the abdomen [4]. In addition, as individual liver lobes cannot be discriminated generally on plain radiographs, it is difficult to accurately localize a foreign body embedded in the liver [14]. In the present case, the foreign body was well detected on plain radiography because of its metallic opacity. Ultrasound and CT provide 

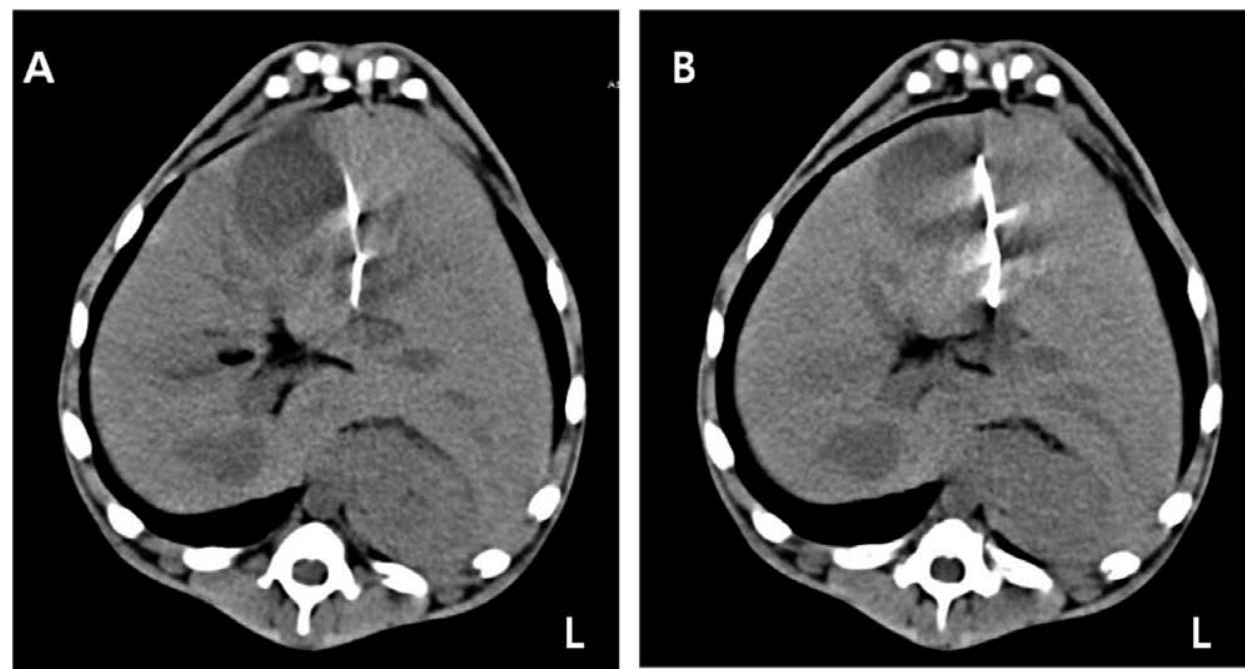

Fig. 3. CT scans of the liver show a linear hyperattenuating material and high contrast artifacts consistent with a metallic foreign body just medial to the gall bladder, and no hepatic parenchymal lesion is apparent.
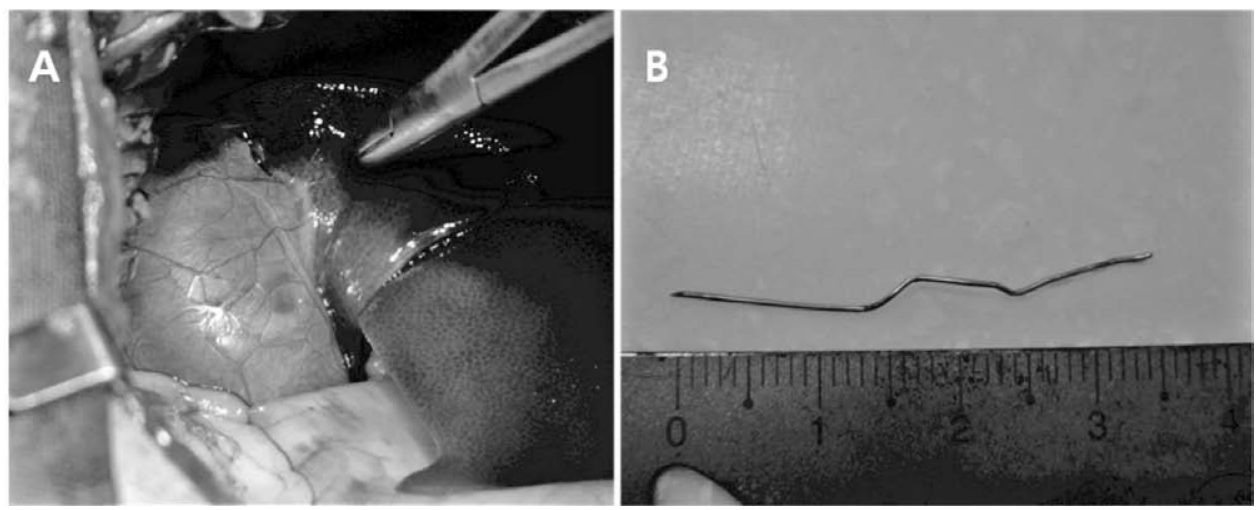

Fig. 4. Operative findings reveal adhesion between the gall bladder wall and medial aspect of the quadrate lobe (A). Gross finding of the wire measuring $3.5 \mathrm{~cm}$ in length (B).

much information associated with the foreign body. Abnormalities of the gall bladder, hepatic parenchyma, bile ducts and hepatic vessels can be identified, and radiolucent foreign bodies can be detected on ultrasound and CT $[2,12,15$, 16]. The reflective quality of a foreign body on ultrasonography is determined based on its acoustic impedance, which varies with physical density $[12,17]$. Metallic foreign bodies produce echogenicity with posterior reverberation $[17$, 18]. In the present case, the foreign body was identified as a hyperechoic linear structure with reverberation corresponding to a metallic foreign body. In humans, hepatic abscess is found as a secondary finding of hepatic foreign body in most cases. However, in the present case, there were no hepatic pathologic changes associated with the foreign body, including gall bladder rupture, hematoma, abscess and necrosis, on ultrasonographic and CT images. In addition, CT scans are essential in confirming the accurate location of a foreign body embedded in the liver [3]. In the present case, we are able to recognize the foreign body's accurate location easily on CT images. We were also able to find some artifacts made by metallic foreign material or contrast media.

Although there has been one case of antibiotic therapy only [2], the recommended treatment is exploratory laparotomy to drain the hepatic abscess and eliminate the foreign body if a hepatic abscess secondary to a hepatic foreign body is detected $[3,9,10,16]$. Despite the benign evolution, the hepatic foreign body in the present case was considered a potentially risk factor for gall bladder rupture or abscess formation because of its location, and it was therefore removed as fast as possible.

This research was supported by the Basic Science Research Program through the National Research Foundation of Korea (NRF) funded by the Ministry of Education, Science and Technology (2010-0007394). 


\section{REFERENCES}

1. Brennan, K.W. and Ihrke, P.J. 1983. Grass awn migration in dogs and cats: a retrospective study of 182 cases. J. Am. Vet. Med. Assoc. 182: 1201-1204.

2. Ching, T.H., Liu, K.L., Lee, Y.C., Chiu, H.M., Lin, J.T. and Wang, H.P. 2006. Sonographic diagnosis of a toothpick traversing the duodenum and penetrating into the liver. J. Clin. Ultrasound. 34: 237-240.

3. Chintamani, Singhal, V., Lubhana, P., Durkhere, R. and Bhandari, S. 2003. Liver abscess secondary to a broken needle migration-a case report. B.M.C. Surg. 7: 3-8.

4. Coulier, B., Tancredi, M.H. and Ramboux, A. 2004. Spiral CT and multidetector-row $\mathrm{CT}$ diagnosis of perforation of the small intestine caused by ingested foreign bodies. Eur. Radiol. 14: 1918-1925.

5. Frendin, J., Funkquist, B., Hansson, K., Lönnemark, M. and Carlsten, J. 1999. Diagnostic imaging of foreign body reactions in dogs with diffuse back pain. J. Small. Anim. Pract. 40: 278 285.

6. Hartley, C., McConnell, J.F. and Doust, R. 2007. Wooden Orbital foreign body in a Weimaraner. Vet. Ophthalmol. 10: 390-393.

7. Hunt, G.B., Worth, A. and Marchevsky, A. 2004. Migration of wooden skewer foreign bodies from the gastrointestinal tract in eight dogs. J. Small. Anim. Pract. 45: 362-367.

8. Jackson, A.H. and Degner, D.A. 2002. Cutaneopulmonary fistula in a dog caused by migration of a toothpick. J. Am. Anim. Hosp. Assoc. 38: 545-547.

9. Kanazawa, S., Ishigaki, K., Miyake, T., Ishida, A., Tabuchi, A., Tanemoto, K. and Tsunoda, T. 2003. A granulomatous liver abscess which developed after a toothpick penetrated the gastrointestinal tract: report of a case. Surg. Today 33: 312314.

10. Karamarkovic, A.R., Djuranovic, S.P., Popovic, N.P., Bum- basirevic, V.D., Sijacki, A.D. and Blazic, I.V. 2007. Hepatic abscess secondary to a rosemary twig migrating from the stomach into the liver. World J. Gastroenterol. 13: 5530-5532.

11. Leskovar, A., Coates, J.R., Russell, K.E., Walker, M.A. and Storts, R.W. 2003. Surgical removal of an intramedullary spinal cord foreign body granuloma in a dog. J. Am. Anim. Hosp. Assoc. 39: 306-310.

12. Matteucci, M.L., Spaulding, K., Dassler, C. and Lee, D. 1999. Ultrasound diagnosis: intra-abdominal wood foreign body. Vet. Radiol. Ultrasound. 40: 513-516.

13. Mukkada, R.J., Chettupuzha, A.P., Francis, V.J., Mathew, P.G., Chirayath, S.P., Koshy, A. and Augustine, P. 2007. Endoscopic removal of chicken bone that caused gastric perforation and liver abscess. Indian J. Gastroenterol. 26: 246-247.

14. Newell, S.M. and Graham, J.P. 2002. The liver and spleen. pp. 540-555. In: Textbook of Veterinary Diagnostic Radiology, 4th ed. (Thrall, D. E. ed.), Saunders, Philadelphia.

15. Nyland, T.G., Mattoon, J.S., Herrgesell, E.J. and Wisner, E.R. 2002. Liver. pp. 93-127. In: Small Animal Diagnostic Ultrasound, 2nd ed. (Nyland, T.G. and Mattoon, J.S. eds.), Saunders, Philadelphia.

16. Santos, S.A., Alberto, S.C., Cruz, E., Pires, E., Figueira, T., Coimbra, E., Estevez, J., Oliveira, M., Novais, L. and Deus, J.R. 2007. Hepatic abscess induced by foreign body: case report and literature review. World J. Gastroenterol. 13: 14661470.

17. Shah, Z.R., Crass, J.R., Oravec, D.C. and Bellon, E.M. 1992. Ultrasonographic detection of foreign bodies in soft tissues using turkey muscle as a model. Vet. Radiol. Ultrasound. 33: 94-100.

18. Wndell, B.A. and Athey, P.A. 1981. Ultrasonic appearance of metallic foreign bodies in parenchymal organs. J. Clin. Ultrasound. 9: 133-135.

19. Wyatt, K.M., Marchevsky, A.M. and Kelly, A. 1999. An enterovesical foreign body in a dog. Aust. Vet. J. 77: 27-29. 